\title{
Small-scale effects on transverse vibrational behavior of single-walled carbon nanotubes with arbitrary boundary conditions
}

\author{
Farzad Ebrahimi* and Parisa Nasirzadeh
}

Department of Mechanical Engineering, Faculty of Engineering, Imam Khomeini International University, Qazvin, Iran

\begin{tabular}{|c|c|}
\hline $\begin{array}{l}\text { ARTICLE INFO } \\
\end{array}$ & A B S T RACT \\
\hline $\begin{array}{l}\text { Article history: } \\
\text { Received September } 6,2014 \\
\text { Accepted } 15 \text { January } 2015 \\
\text { Available online } \\
\text { 16 January } 2015 \\
\text { Keywords: } \\
\text { Free vibration } \\
\text { Nanobeam } \\
\text { Eringen nonlocal elasticity theory }\end{array}$ & $\begin{array}{l}\text { In the present paper, the differential transformation method is employed to develop a semi- } \\
\text { analytical solution for free transverse vibration of single-walled carbon nanotube (SWCNT) } \\
\text { with arbitrary boundary conditions. The small scale effect is taken into consideration via } \\
\text { Eringen's nonlocal elasticity theory while the transverse shear deformation effects and rotary } \\
\text { inertia are taken into account in presented Timoshenko beam theory. Through variational } \\
\text { formulation and the Hamilton's principle the governing differential equations and the boundary } \\
\text { conditions are derived and then solved by a semi-analytical method called differential } \\
\text { transformation method (DTM) for various frequency modes of beams and different edge } \\
\text { conditions. Comparisons made between the present results and results reported by well-known } \\
\text { references for special cases treated before, have confirmed accuracy and efficiency of the } \\
\text { presented approach. The effects of several parameters such as transverse shear deformation } \\
\text { effects, slenderness ratios, boundary conditions and small scale on vibration characteristics of } \\
\text { SWCNT are examined. The present study illustrates that the vibration characteristics of an } \\
\text { SWCNT are strongly dependent on the small scale parameters. }\end{array}$ \\
\hline
\end{tabular}

(C) 2015 Growing Science Ltd. All rights reserved.

\section{Introduction}

The differential transformation method is an efficient and useful numerical method for the rapid solutions of linear and non-linear partial differential equations. It is an alternative discrete approach to directly solve the governing equations of various engineering problems. This method first proposed by Zhou (1986) in solving linear and non-linear initial value problems in electrical circuit analysis. Due to its simplicity and accuracy, DTM has been widely employed in many areas of industry and mathematics. Several researchers have addressed the linear and nonlinear static and dynamic problems of beams and plates by DTM but its application in micro- and nano-scale beams and tubes problems is scarce.

* Corresponding author

E-mail addresses: febrahimy@eng.ikiu.ac.ir (F. Ebrahimi) 
Nanoscale engineering materials have significant mechanical, electrical and thermal performances that are superior to the conventional structural materials. They have attracted great interest in modern science and technology after the invention of carbon nanotubes (CNTs) by Iijima (1991). For example, in micro/nano electromechanical systems (MEMS/NEMS); nanostructures have been used in many areas including communications, machinery, information technology, biotechnology technologies. So far, three main methods were provided to study the mechanical behaviors of nanostructures: atomistic model (Baughman et al., 2002), semi-continuum (Li \& Chou, 2003) and continuum models (Wang and Cai, 2006). However, both atomistic and semi-continuum models are computationally expensive and are not suitable for analyzing large scale systems. In other words, since conducting experiments at the nanoscale is a daunting task, and atomistic modeling is restricted to small-scale systems owing to computer resource limitations, continuum mechanics offers an easy and useful tool for the analysis of CNTs. Therefore, there are considerable efforts made to develop and calibrate continuum structural models for CNTs analysis and these efforts have been summarized in the previous papers.

Moreover due to the inherent size effects, at nanoscale, the mechanical characteristics of nanostructures are often significantly different from their behavior at macroscopic scale. Such effects are essential for nanoscale materials or structures and the influence on nano-instruments is great (Maranganti and Sharma, 2007). Generally, theoretical studies on size effects at nanoscale are by means of surface effects (Zhu et al., 2009), strain gradients in elasticity (Mindlin, 1964) and plasticity (Aifantis, 1984), and the nonlocal stress field theory (Eringen, 1983; 1972a). Unfortunately, the classical continuum theories are deemed to fail for these nanostructures, because the length dimensions at nano scale are often sufficiently small such that call the applicability of classical continuum theories into the question. Consequently, the classical continuum models need to be extended to consider the nanoscale effects and this can be achieved through the nonlocal elasticity theory proposed by Eringen (Eringen, 1972a) which considers the size-dependent effect. According to this theory, the stress state at a reference point is considered as a function of strain states of all points in the body. This nonlocal theory is proved to be in accordance with atomic model of lattice dynamics and with experimental observations on phonon dispersion (Eringen, 1983). In nonlocal theory, the nonlocal nanoscale in the constitutive equation could be considered simply as a material-dependent parameter.

In recent years, nanobeams and carbon nanotubes hold a wide variety of potential applications (Zhang et al., 2004; Wang, 2005; Wang and Varadan, 2006) such as sensors, actuators, transistors, probes, and resonators in NEMSs. Thus, establishing an accurate model of nanobeams is a key issue for successful NEMS design. As a result, nanotechnological research on vibrational properties of nanobeams is important because such components can be used as design components in nano-sensors and nano-actuators. Furthermore, many researchers worked on bending, buckling and vibration of beam-like elements in nanoscale (Peddieson et al., 2003; Liew et al., 2008; Amara et al., 2010) in most of which the nonlocal Euler-Bernoulli beam theory has been employed for vibration analysis of nanobeams (Lu et al., 2006; Zhang et al., 2005; Xu, 2006). Samaei and Mirsayar (2011) considered the small scale effects on buckling characteristics of multi-walled CNTs. Kiani (2010) presented a meshless approach for free transverse vibration analysis of embedded SWCNTs with arbitrary boundary conditions accounting for nonlocal effect. Also But since this theory does not account for transverse shear and rotary inertia effects, it gives unreliable results and overpredicts the vibration characteristics for stubby beams and especially for higher frequencies of vibration because the effect of transverse shear deformation become significant and cannot be neglected (Wang et al., 2000). Employing nonlocal Timoshenko beam model Ansari and Ramezannezhad studied the large-amplitude vibrations of embedded multi-walled CNTs including thermal effects (Ansari \& Ramezannezhad, 2011). Kiani and Mehri (2010) examined the behaviour of nanotube structures under a moving nanoparticle using nonlocal beam theories. Besides, Torabi and Nafar Dastgerdi (2012) presented an analytical method for free vibration analysis of Timoshenko beam theory applied to cracked nanobeams using a nonlocal elasticity model. While Ansari et al. (2011) presented a sixth-order compact finite difference method for vibrational analysis of nanobeams embedded in an elastic medium based on nonlocal beam theory. 
Ghorbanpourarani et al. (2010) considered the cylindrical shell and beam models to study transverse vibration of short CNTs. Recently Ansari and Sahmani (2012) investigated the small scale effect on vibrational response of SWCNTs with different boundary conditions based on nonlocal beam models. Most recently Kiani (2013) presented the vibration analysis of elastically restrained double-walled

CNTs on elastic foundation subjected to axial load using nonlocal shear deformable beam theories. Motivated by these considerations, in this study, differential transformation method is applied in analyzing vibration characteristics of SWCNTs under various edge conditions. The superiority of the DTM is its simplicity and good precision and depends on Taylor series expansion while it takes less time to solve polynomial series. It is different from the traditional high order Taylor's series technique, which needs symbolic competition of the necessary derivatives of the data functions. The Taylor series method is computationally taken long time for large orders. With this technique, it is possible to reach highly reliable results or even exact solutions for differential equations. With this method, the given partial differential equation and related initial conditions are transformed into a recurrence equation, which could eventually lead to the solution of a system of algebraic equations as coefficients of a power series solution. This helps obtain exact and approximate solutions of linear and nonlinear ordinary and partial differential equations and there is no necessary for linearization or perturbations, large computational work and round-off errors are avoided. It's a proper technique to analyze beam vibrations. To the author's best knowledge there is no work reported on the application of DTM on vibration analysis of SWCNTs based on nonlocal Timoshenko bema model. In this study, the governing equations and boundary conditions for the free vibration of a nonlocal Timoshenko beam have been extracted via Hamilton principle. Unlike the Euler beam model, the Timoshenko beam model helps for the effect of transverse shear deformation, which becomes significant for CNTs with small length-todiameter ratios. The detailed mathematical derivations are presented and numerical investigations are performed while the emphasis is placed on investigating the impact of different parameters such as transverse shear deformation effects, slenderness ratios, boundary conditions and small scale on vibration characteristics of SWCNT. Comparisons with the results from the existing literature are provided and the good agreement between the results of the proposed method of this paper and those available in literature validated the presented approach. Numerical results are presented to serve as benchmarks for the application and the design of nanoelectronic and nano-drive devices, nanooscillators, and nanosensors, in which CNTs act as basic elements.

\section{Brief statement of the differential transformation method}

Differential transformation technique is one of the useful techniques to solve the differential solutions with small calculation errors and ability to solve nonlinear equations with boundary conditions value problems. Abdel-Halim Hassan (2002) used the DTM on eigenvalues and normalized eigenfunctions. In addition, Wang (2013) presented the axial vibration analysis of stepped bars utilizing DTM. The DTM is proved to be an appropriate computational tool for different engineering problems. Using differential transformation technique, the ordinary and partial differential equations can be transformed into algebraic equations, from which a closed-form series solution can be obtained easily. In this method, certain transformation rules are used to both the governing differential equations of motion and the boundary conditions of the system in order to transform them into a set of algebraic equations as presented in Table 1 and Table 2. The solution of these algebraic equations gives the desired results of the problem. The basic definitions and the application procedure of this method can be introduced as follows:

The transformation equation of function $\mathrm{f}(\mathrm{x})$ can be defined as (Chen and $\mathrm{Ju}, 2004)$ :

$$
F[k]=1 / k !\left(\frac{d^{k} f(x)}{d x^{k}}\right)_{x=x_{0}}
$$


where $\mathrm{f}(\mathrm{x})$ is the original function and $\mathrm{F}[\mathrm{k}]$ is the transformed function. The inverse transformation is defined as:

$$
f(x)=\sum_{k=0}^{\infty}\left(x-x_{0}\right)^{k} F[k]
$$

Table 1. Some of the transformation rules of the one-dimensional DTM (Chen and Ju, 2004)

Original function

$$
\begin{array}{cc}
f(x)=g(x) \pm h(x) & F(K)=G(K) \pm H(K) \\
f(x)=\lambda g(x) & F(K)=\lambda G(K) \\
f(x)=g(x) h(x) & F(K)=\sum_{l=0}^{K} G(K-l) H(l) \\
f(x)=\frac{d^{n} g(x)}{d x^{n}} & F(K)=\frac{(k+n) !}{k !} G(K+n) \\
f(x)=x^{n} & F(K)=\delta(K-n)=0 \quad \text { if } \mathrm{k} \neq \mathrm{n} \\
&
\end{array}
$$

Table 2. Transformed boundary conditions (B.C.) based on DTM (Chen and Ju, 2004)

\begin{tabular}{cccc}
\hline \multicolumn{1}{l}{ X $=0$} & & $\mathrm{X}=1$ \\
\hline Original B.C. & Transformed B.C. & Original B.C. & Transformed B.C. \\
\hline$f(0)=0$ & $F[0]=0$ & $f(1)=0$ & $\sum_{k=0}^{\infty} F[k]=0$ \\
$\frac{d f}{d x}(0)=0$ & $F[1]=0$ & $\frac{d f}{d x}(1)=0$ & $\sum_{k=0}^{\infty} k F[k]=0$ \\
$\frac{d^{2} f}{d x^{2}}(0)=0$ & $F[2]=0$ & $\frac{d^{2} f}{d x^{2}}(1)=0$ & $\sum_{k=0}^{\infty} k(k-1) F[k]=0$ \\
$\frac{d^{3} f}{d x^{3}}(0)=0$ & $F[3]=0$ & $\frac{d^{3} f}{d x^{3}}(1)=0$ & $\sum_{k=0}^{\infty} k(k-1)(k-2) F[k]=0$ \\
\hline
\end{tabular}

Combining Eq. (1) and Eq. (2) one obtains:

$$
f(x)=\sum_{k=0}^{\infty} \frac{\left(x-x_{0}\right)^{k}}{k !}\left(\frac{d^{k} f(x)}{d x^{k}}\right)_{x=x_{0}}
$$

In actual application, the function $\mathrm{f}(\mathrm{x})$ is expressed by a finite series and Eq. (3) can be written as follows:

$$
f(x)=\sum_{k=0}^{n} \frac{\left(x-x_{0}\right)^{k}}{k !}\left(\frac{d^{k} f(x)}{d x^{k}}\right)_{x=x_{0}}
$$

which implies that the term in relation (5) is negligible.

$$
f(x)=\sum_{k=n+1}^{\infty} \frac{\left(x-x_{0}\right)^{k}}{k !}\left(\frac{d^{k} f(x)}{d x^{k}}\right)_{x=x_{0}}
$$




\section{Nonlocal Timoshenko beam equations and boundary conditions}

Consider a beam with length L and the cross sectional area of A. Based on Timoshenko beam theory, strain-displacement and strain energy relations are as follows (Wang et al., 2000):

$$
\begin{aligned}
& \varepsilon_{x x}=z \frac{d \emptyset}{d x} \\
& \gamma_{x z}=\emptyset+\frac{d w}{d x} \\
& U=1 / 2 \int_{0}^{L} \int_{A}\left(\sigma_{x x} \varepsilon_{x x}+\sigma_{x z} \gamma_{x z}\right) d A d x
\end{aligned}
$$

where $x$ is the longitudinal coordinate measured from the left end of the beam and the $z$ is the coordinate measured from the mid-plane of the beam, $w$ represents the lateral deflection and $\varnothing$ is the bending slope of the beam due to bending and $\varepsilon_{\mathrm{xx}}$ is the normal strain and $\gamma_{\mathrm{xz}}$ is the transverse shear strain and $\mathrm{U}$ represents the strain energy. $\sigma_{\mathrm{xx}}$ and $\sigma_{\mathrm{xz}}$ are normal stress and the transverse shear stress respectively. After substituting Eq. (5) and Eq. (7) in Eq. (8) and putting bending moment and shear force in Eq. (8), strain energy is written as:

$$
\begin{aligned}
& U=1 / 2 \int_{0}^{L} \int_{A}\left(\sigma_{x x} z \frac{d \emptyset}{d x}+\sigma_{x z}\left(\varnothing+\frac{d w}{d x}\right)\right) d A d x=1 / 2\left(M \frac{d \emptyset}{d x}+Q\left(\varnothing+\frac{d w}{d x}\right)\right) d x \\
& M=\int_{A} \sigma_{x x} z d A \\
& Q=\int_{A} \sigma_{x z} d A
\end{aligned}
$$

where $\mathrm{M}$ and $\mathrm{Q}$ are bending moment and the shear force respectively. The kinetic energy $\mathrm{T}$, by assuming free harmonic motion and rotary inertia effect, is written as:

$$
T=1 / 2 \int_{0}^{L}\left(\rho A \omega^{2} w^{2}+\rho I \omega^{2} \emptyset^{2}\right) d x
$$

where $\omega$ is the circular frequency of vibration and $\rho$ and I are the mass density and the second moment of area of the beam respectively. Applying Hamilton's principle (Chow, 2013) requires:

$$
\delta(T-U)=0=\int_{0}^{L}\left(-M \frac{d \delta \emptyset}{d x}-Q\left(\delta \varnothing+\frac{d \delta w}{d x}\right)+\rho A \omega^{2} w \delta w+\rho I \omega^{2} \emptyset \delta \emptyset\right) d x
$$

and after performing integration by parts, we reach:

$$
0=\int_{0}^{L}\left[\left(\frac{d M}{d x}-Q+\rho I \omega^{2} \emptyset\right) \delta \emptyset+\left(\frac{d Q}{d x}+\rho A \omega^{2} w\right) \delta w\right] d x-[M \delta \varnothing]_{0}^{L}-[Q \delta w]_{0}^{L}
$$

This results in the following equations:

$$
\begin{aligned}
& \frac{d M}{d x}=Q-\rho I \omega^{2} \emptyset \\
& \frac{d Q}{d x}=-\rho A \omega^{2} w
\end{aligned}
$$

and the boundary conditions are in two forms as below relations: 
Either $\mathrm{w}$ or $\mathrm{Q}=0$

Either $\varnothing$ or $M=0$

As it shows the equations appear to be the same as local Timoshenko beam theory, but the shear force and bending moment expressions for nonlocal beam theory must be different. The constitutive equation of classical elasticity is an algebraic relationship between stress and strain tensors while Eringen nonlocal elasticity includes spatial integrals which indicate the average effect of strain of all points of the body to the stress tensor at the given point (Eringen, 1972b; 1983). Since the spatial integrals in constitutive equations are mathematically difficult to solve, they can be converted to the equal differential constitutive equations under certain conditions. The nonlocal constitutive stress-strain relation for an elastic material in the one dimensional case beam can be simplified as (Eringen, 1983):

$$
\sigma_{x x}-\left(e_{0} a\right)^{2} \frac{d^{2} \sigma_{x x}}{d x^{2}}=E \varepsilon_{x x}
$$

where $E$ is the young modulus, $e_{0} a$ is the scale coefficient that incorporates the small scale effect and a represents the internal characteristic length and $e_{0}$ is the constant appropriate to each material which is measured experimentally. The local and nonlocal constitutive shear strain-stress relations are the same, since the form of Eringen nonlocal constitutive model cannot be applied in $\mathrm{z}$ direction:

$$
\sigma_{x z}=G \gamma_{x z}
$$

where $G$ is the shear modulus. After multiplying the term (zdA) and integration over the area A Eq. (14) becomes:

$$
M-\left(e_{0} a\right)^{2} \frac{d^{2} M}{d x^{2}}=E I \frac{d \emptyset}{d x}
$$

Integrating Eq. (15) over the area yields the following relation:

$$
Q=K_{s} G A\left(\varnothing+\frac{d w}{d x}\right)
$$

where $\mathrm{K}_{\mathrm{s}}$ is the shear correction factor that accounts for the difference in the constant state of shear stress in the Timoshenko beam theory and the parabolic variation of the actual shear stress through the depth of the cross section (Wang et al. 2000). Now by substituting Eqs. (15-16) in Eq. (21) the moment can be reached as follows:

$$
M=E I \frac{d \emptyset}{d x}-\left(e_{0} a\right)^{2}\left(\rho A \omega^{2} w+\rho I \omega^{2} \frac{d \emptyset}{d x}\right)
$$

and by utilizing equations (22) and (23) in Timoshenko beam equations (15) and (16) the governing equation for the vibration of nonlocal Timoshenko beam may be obtained as:

$$
\begin{aligned}
& E I \frac{d^{2} \emptyset}{d x^{2}}-K_{S} G A\left(\emptyset+\frac{d w}{d x}\right)+\rho I \omega^{2} \emptyset-\left(e_{0} a\right)^{2}\left(\rho A \omega^{2} \frac{d w}{d x}+\rho I \omega^{2} \frac{d^{2} \emptyset}{d x^{2}}\right)=0 \\
& K_{S} G A\left(\frac{d \emptyset}{d x}+\frac{d^{2} w}{d x^{2}}\right)+\rho A w \omega^{2}=0
\end{aligned}
$$

On the basis of Eq. (17) and Eq. (18) and due to various ending conditions of the beam e.g. for a simply supported end as: 


$$
w=0 \quad, \quad M=E I \frac{d \emptyset}{d x}-\left(e_{0} a\right)^{2}\left(\rho A \omega^{2} w+\rho I \omega^{2} \frac{d \emptyset}{d x}\right)=0
$$

and for a clamped end as:

$$
w=0, \varnothing=0
$$

and for a free end as:

$$
M=E I \frac{d \emptyset}{d x}-\left(e_{0} a\right)^{2}\left(\rho A \omega^{2} w+\rho I \omega^{2} \frac{d \emptyset}{d x}\right)=0, \quad Q=K_{S} G A\left(\emptyset+\frac{d w}{d x}\right)=0
$$

\section{Non-Dimensional parameters}

The non-dimensional parameters contributes to simplify the equations and to make comparisons in the studies possible. The non-dimensional parameters are introduced as following terms:

$$
\begin{array}{ll}
\bar{x}=\frac{x}{L} ; & \bar{W}=\frac{w}{L} \\
\lambda^{2}=\omega^{2} \frac{\rho A L^{4}}{E I}=\text { frequency parameter; } & \Omega=\frac{E I}{K_{S} G A L^{2}}=\text { shear deformation parameter } \\
\propto=\frac{\mathrm{e}_{0} \mathrm{a}}{\mathrm{L}}=\text { scaling effect parameter; } & \varepsilon=\frac{\mathrm{L} \sqrt{\mathrm{A}}}{\sqrt{\mathrm{I}}}=\text { slenderness ratio } \\
\hline
\end{array}
$$

By applying the non-dimensional parameters to the governing Eq. (24) and Eq. (25) the following relations obtained:

$$
\begin{aligned}
& \Omega\left(1-\frac{\alpha^{2} \lambda^{2}}{\varepsilon^{2}}\right) \frac{d^{2} \bar{\emptyset}}{d \bar{x}^{2}}+\left(\frac{\Omega \lambda^{2}}{\varepsilon^{2}}-1\right) \emptyset-\left(\alpha^{2} \lambda^{2} \Omega+1\right) \frac{d \bar{w}}{d \bar{x}}=0 \\
& \left(\frac{d \emptyset}{d \bar{x}}+\frac{d^{2} \bar{w}}{d \bar{x}^{2}}\right)+\lambda^{2} \Omega \bar{w}=0
\end{aligned}
$$

Also boundary conditions equations (26)- (28) appear for a simple supported end as:

$$
\bar{w}=0, M=\left(\Omega-\frac{\Omega \alpha^{2} \lambda^{2}}{\varepsilon^{2}}\right) \frac{d \emptyset}{d \bar{x}}-\Omega \alpha^{2} \lambda^{2} \bar{w}=0
$$

And for a clamped end as:

$$
\bar{w}=0, \varnothing=0
$$

And for a free end as:

$$
M=\left(\Omega-\frac{\Omega \alpha^{2} \lambda^{2}}{\varepsilon^{2}}\right) \frac{d \emptyset}{d \bar{x}}-\Omega \alpha^{2} \lambda^{2} \bar{w}=0 ; Q=K_{S} G A\left(\varnothing+\frac{d \bar{w}}{d \bar{x}}\right)=0
$$

\section{Solution with differential transformation method}

As stated in section 2 the differential transform method, certain transformation rules are defined and applied to the governing differential equations and their relevant boundary conditions to transform them into a set of algebraic equations. Solution of these algebraic equations gives the desired solution 
of the problem. According to the basic transformation operations introduced in Table 1, the transformed form of the governing Eqs. (29-20) may be obtained as:

$$
\begin{aligned}
& \Omega\left(1-\frac{\alpha^{2} \lambda^{2}}{\varepsilon^{2}}\right)(K+1)(K+2) \phi(K+2)+\left(\frac{\Omega \lambda^{2}}{\varepsilon^{2}}-1\right) \phi(K)-\left(\alpha^{2} \lambda^{2} \Omega+1\right)(K+1) \bar{W}(K+1)=0 \\
& (K+1) \phi(K+1)+(K+1)(K+2) \bar{W}(K+2)+\lambda^{2} \Omega \bar{W}(K)=0
\end{aligned}
$$

The transformed form of boundary conditions is presented in Table 2 . The frequency equations may be derived by incorporating the transformed boundary conditions simultaneously. As it has been mentioned before, three kinds of edge conditions are considered in the present research: free, simply supported, and clamped. It shall be noted that $\mathrm{W}(\mathrm{k})$ and $\Phi(\mathrm{k})$ are transforms of $\mathrm{w}(\mathrm{x})$ and $\phi(\mathrm{x})$, respectively. By substituting values for $\mathrm{k}=0,1,2, \ldots, \alpha=0, \varepsilon=34.641$ and $\Omega=0.2436$ in equations (34) and (35), we can evaluate the amounts of $W(2), W(3), \ldots$ and $\Phi(2), \Phi(3), \ldots$ in terms of $\omega^{2}$ and some constants like $c_{1}, \ldots$. The values can be achieved with a computer program and after substituting W(i) and $\Phi(\mathrm{i})$ in boundary conditions the following equation may be obtained:

$$
N_{r 1}^{(n)}(\omega) c_{1}+N_{r 2}^{(n)}(\omega) c_{2}=0 \quad r=1,2, \ldots, n
$$

Here $N s$ are polynomials in terms of $\omega$ corresponding to $n^{\text {th }}$ term. When solving the equation (36) in matrix form and studying the Existence condition of the non-trivial solutions yields the following characteristic determinant:

$$
\left|\begin{array}{ll}
N_{11}^{n}(\omega) & N_{12}^{n}(\omega) \\
N_{21}^{n}(\omega) & N_{22}^{n}(\omega)
\end{array}\right|=0
$$

which may be used to calculate the dimensionless frequencies. The solution of Eq. (37) gives $\omega_{\mathrm{r}}^{\mathrm{n}}$ which is the $\mathrm{r}^{\text {th }}$ estimated dimensionless natural frequency for $n$th iteration. To determine the value of the $n$th natural frequency, the following convergence criterion may be used:

$$
\frac{\left|\omega_{r}^{n}-\omega_{r}^{n-1}\right|}{\left|\omega_{r}^{n}\right|}<\delta
$$

where $n$ is the iteration counter, and $\delta$ is a sufficiently small number that is chosen as $\delta=0.0001$ in the present study. With respect to the differential transformation method, an algorithm in MATLAB software has been developed in order to determine the vibration characteristics of the nonlocal Timoshenko nanobeam.

\section{Results and Discussion}

In the present study the impact of small scale coefficient as well as the effect of slenderness on first, second and third frequencies of the SWCNTs have been studied. Also three types of boundary conditions e.g. free simply supported, and clamped ends are compared. As a validation example, the first three natural frequencies of nonlocal Timoshenko beam with clamped-clamped ends, Clampedsimply supported and simply supported -Free edge conditions already studied analytically by Wang et al. (2007) are reexamined. The mechanical properties of the nonlocal Timoshenko nanobeam are considered as presented Table 3. Also the Timoshenko shear correction factor $\left(k_{s}\right)$ is taken as 0.563 . Table 4 compares the first three nondimensional frequency obtained by the present method with the results of Wang et al. (2007) for nonlocal nanobeam with both clamped ends and $\mathrm{L} / \mathrm{d}=10$. 
Table 3. Mechanical properties of nonlocal Timoshenko nanobeam (Wang et al., 2007)

\begin{tabular}{ccc}
\hline property & Unit & \\
\hline $\mathrm{E}$ & $\mathrm{TPa}$ & 5.5 \\
$\mathrm{P}$ & $\mathrm{g} . \mathrm{cm}^{-3}$ & 2.3 \\
$v$ & - & 0.19 \\
\hline
\end{tabular}

Table 4. First three nondimensional frequency $\sqrt{\lambda}$ of nonlocal Timoshenko beam for both clamped ends and $\mathrm{L} / \mathrm{d}=10$

\begin{tabular}{|c|c|c|c|c|c|c|c|c|c|}
\hline & \multicolumn{3}{|c|}{ Mode 1} & \multicolumn{3}{|c|}{ Mode 2} & \multicolumn{3}{|c|}{ Mode 3} \\
\hline$\alpha$ & Present & $\begin{array}{c}\text { Wang et al. } \\
(2007)\end{array}$ & Diff. \% & Present & $\begin{array}{c}\text { Wang et al. } \\
(2007)\end{array}$ & Diff. $\%$ & Present & $\begin{array}{c}\text { Wang et al. } \\
(2007)\end{array}$ & Diff. \% \\
\hline 0 & 4.530 & 4.450 & 1.76 & 7.19 & 6.95 & 3.33 & 9.62 & 9.21 & 4.1 \\
\hline 0.1 & 4.423 & 4.3471 & 1.72 & 6.67 & 6.495 & 2.62 & 8.42 & 8.22 & 2.38 \\
\hline 0.3 & 3.830 & 3.7895 & 1.05 & 4.99 & 4.942 & 1.14 & 5.95 & 5.84 & 1.74 \\
\hline 0.5 & 3.265 & 3.242 & 0.72 & 3.999 & 3.99 & 0.15 & 4.75 & 4.67 & 1.53 \\
\hline 0.7 & 2.850 & 2.8383 & 0.41 & 3.45 & 3.41 & 0.89 & 4.05 & 3.99 & 1.33 \\
\hline
\end{tabular}

For calculating the exact difference between the results of present and available results in literature, relation Eq. (39) has been applied:

$$
\% \text { difference }=100 \times \frac{\mid \text { refrence }- \text { present } \mid}{\text { present }}
$$

In addition, Table 5 compares the first three nondimensional frequency of nonlocal nanobeam for two kinds of boundary conditions (Clamped-Simple and Simple-Free) and L/d=10 obtained by the present method with the results of Wang et al. (2007). As can be seen in Tables 4 and Table 5 the good agreement and a close correlation among the results validate the proposed method of solution.

Moreover, the convergence of the differential transformation method is perused. In Fig. 1 the convergence of the third frequency of a nonlocal Timoshenko beam with both clamped ends is presented. It illustrates that the third frequency converges at 46th iteration, while the first and the second frequencies have been converged before, in this example at $29^{\text {th }}$ and $37^{\text {th }}$ iterations.

Table 5. First nondimensional natural frequency $\sqrt{\lambda}$ of nonlocal Timoshenko beam for two kinds of boundary conditions and $\mathrm{L} / \mathrm{d}=10$.

\begin{tabular}{lcccccc}
\hline & \multicolumn{3}{c}{ Clamped-Simple } & \multicolumn{3}{c}{ Simple-Free } \\
\hline$\alpha$ & Present & $\begin{array}{c}\text { Wang et al. } \\
(2007)\end{array}$ & Diff. \% & Present & $\begin{array}{c}\text { Wang et al. } \\
(2007)\end{array}$ & Diff. \% \\
\hline 0 & 3.82 & 3.7845 & 0.929 & 3.08 & 3.0929 & 0.418 \\
0.1 & 3.73 & 3.6939 & 0.967 & 3.059 & 3.0243 & 1.13 \\
0.3 & 3.23 & 3.2115 & 0.5727 & 2.91 & 2.6538 & 8.8 \\
0.7 & 2.415 & 2.4059 & 0.37 & 2.4 & 2.0106 & 16 \\
\hline
\end{tabular}

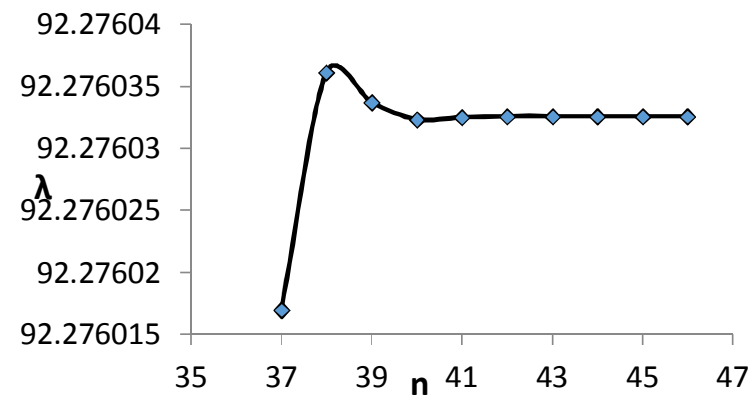

Fig. 1. Convergence of third frequency, $\mathrm{L} / \mathrm{d}=10, \alpha=0$

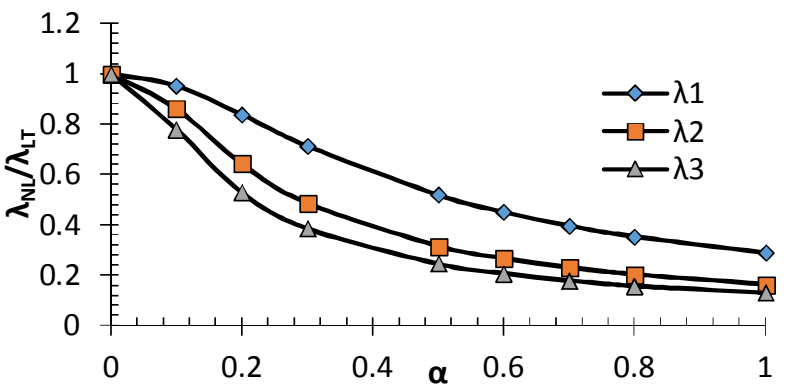

Fig. 2. Effect of small scale on different frequency modes, $\mathrm{L} / \mathrm{d}=10$, clamped ends 


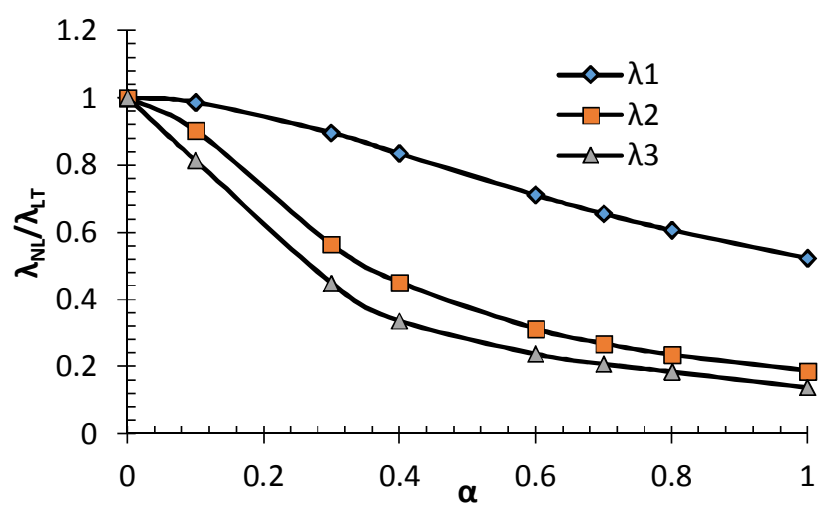

Fig. 3. Effect of small scale on frequency ratio for different frequency modes, $L / d=10$, simply supported beam

The variables in the governing Eq. (24) and Eq. (25) are $\alpha, \varepsilon$ and $\Omega$. $\alpha$ relates to the small scale effect, $\varepsilon$ is in terms of slenderness $(\mathrm{L} / \mathrm{d})$ and $\Omega$ relates to the mechanical properties and slenderness. So it is possible to investigate the effects of slenderness and small scale on various frequencies and mode shapes of a nonlocal Timoshenko beam. Furthermore, determination of the magnitude of $\mathrm{e}_{0}$ is significant due to its prominent effect on small scale coefficient. Some researchers worked on estimating the magnitude of $\mathrm{e}_{0}$ a. For instance, Zhang et al. (2005) estimated the magnitude of the parameter for CNTs approximately 0.82 . In this study we adopt $0 \leq \alpha<0.8$ in our investigations as reported by Lu et al. (2006).

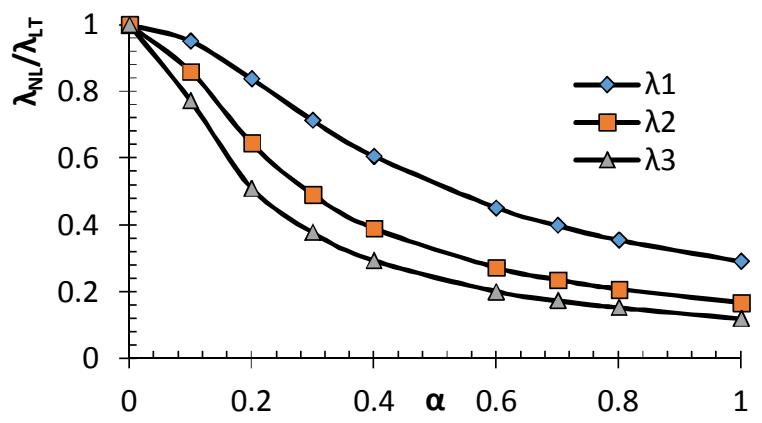

Fig. 4. Effect of small scale on frequency ratio for different frequency modes, $\mathrm{L} / \mathrm{d}=10$, clamped-simply beam

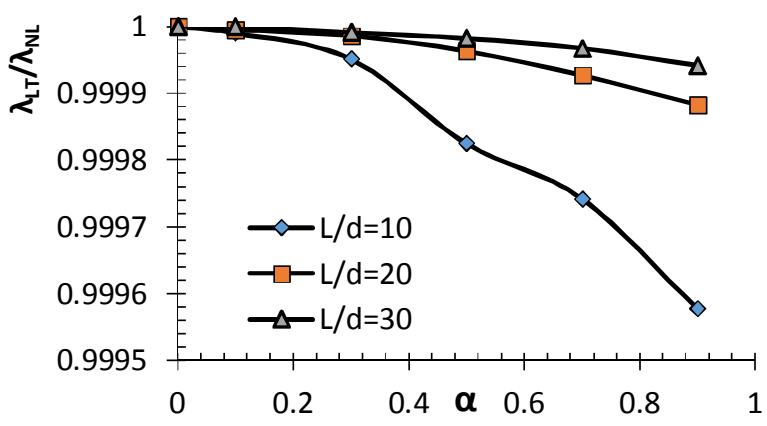

Fig. 5. Small scale effect on frequency ratio with different values of $\mathrm{L} / \mathrm{d}$, both ends clamped

As the Figs. (2-4) show when the coefficient $\alpha$ equals zero, the frequency of nonlocal Timoshenko beam equals its local counterpart. As the coefficient increases the frequency ratio decreases, which means the nonlocal beam frequency become smaller than the local counterparts. This reduction is especially noticeable in higher modes and cannot be neglected. In sum, the small scale effect makes the beam more flexible since in nonlocal theory elastic springs link atoms together (Liew et al., 2008).

Fig. 5 indicates that the small scale have significant effect on short beams and as the beam gets longer its impact gradually become negligible. So the small scale will diminish for a very long and thin (slender) beam. The effects of slenderness of the nanobeam have been presented in Table 6 . In this table the first three frequencies of nonlocal Timoshenko beam with two kinds of boundary conditions (Simply supported- Simply supported and clamped-simply supported) has been presented for various values of length to diameter ration of the SWCNT. The effect of small scale parameter on first three frequency ratios with different values of $\mathrm{L} / \mathrm{d}$, for the case of clamped-simply supported beam has been illustrated in Fig. 6. It is also noticeable that the small scale has significant effect on short beams and as the beam gets longer its impact gradually become negligible. Fig. 7 illustrates that the nonlocal 
Timoshenko beam frequency approaches the local Timoshenko beam frequency as the slenderness increases.

Table 6. First three frequencies $\sqrt{\lambda}$ of nonlocal Timoshenko beam with two kinds of boundary conditions

\begin{tabular}{|c|c|c|c|c|}
\hline \multirow[b]{2}{*}{$e_{0} a$} & \multirow[b]{2}{*}{ Mode } & $\mathrm{L} / \mathrm{d}=10$ & $\mathrm{~L} / \mathrm{d}=20$ & $\mathrm{~L} / \mathrm{d}=3$ \\
\hline & & \multicolumn{3}{|c|}{ Simply supported- Simply supported beam } \\
\hline 0 & 1 & 3.08057 & 3.12577 & 3.13451 \\
\hline 0.1 & 1 & 3.08056 & 3.12577 & 3.13451 \\
\hline 0.3 & 1 & 3.08056 & 3.12577 & 3.1345 \\
\hline 0.5 & 1 & 3.08052 & 3.12576 & 3.1345 \\
\hline 0.7 & 1 & 3.08047 & 3.12574 & 3.13449 \\
\hline 0.9 & 1 & 3.0804 & 3.12572 & 3.13448 \\
\hline 0 & 2 & 5.94588 & 6.18907 & 6.24037 \\
\hline 0.1 & 2 & 5.94584 & 6.18906 & 6.24036 \\
\hline 0.3 & 2 & 5.94558 & 6.18898 & 6.24033 \\
\hline 0.5 & 2 & 5.94466 & 6.18882 & 6.24025 \\
\hline 0.7 & 2 & 5.94425 & 6.18858 & 6.24015 \\
\hline 0.9 & 2 & 5.94318 & 6.18826 & 6.24000 \\
\hline 0 & 3 & 8.53236 & 9.15198 & 9.29787 \\
\hline 0.1 & 3 & 8.53225 & 9.15194 & 9.29785 \\
\hline 0.3 & 3 & 8.53139 & 9.15165 & 9.29771 \\
\hline 0.5 & 3 & 8.52995 & 9.15107 & 9.29743 \\
\hline 0.7 & 3 & 8.52936 & 9.1502 & 9.29702 \\
\hline \multirow[t]{2}{*}{0.9} & 3 & 8.52366 & 9.14904 & 9.29647 \\
\hline & & \multicolumn{3}{|c|}{$\mathrm{L} / \mathrm{d}=10$} \\
\hline $\mathrm{e}_{0} \mathrm{a}$ & Mode & \multicolumn{3}{|c|}{ Clamped-simply supported beam } \\
\hline 0 & 1 & 3.829744 & 3.901179 & 3.915187 \\
\hline 0.1 & 1 & 3.829726 & 3.901175 & 3.915186 \\
\hline 0.3 & 1 & 3.829653 & 3.901155 & 3.915176 \\
\hline 0.5 & 1 & 3.829491 & 3.901111 & 3.915155 \\
\hline 0.7 & 1 & 3.829248 & 3.901045 & 3.915136 \\
\hline 0.9 & 1 & 3.828925 & 3.900957 & 3.915086 \\
\hline 1 & 1 & 3.828732 & 3.900904 & 3.915063 \\
\hline 0 & 2 & 6.644277 & 6.948166 & 7.01359 \\
\hline 0.1 & 2 & 6.644219 & 6.948148 & 7.013581 \\
\hline 0.3 & 2 & 6.642754 & 6.948008 & 7.013516 \\
\hline 0.5 & 2 & 6.642824 & 6.947726 & 7.013386 \\
\hline 0.7 & 2 & 6.641431 & 6.947305 & 7.013191 \\
\hline 0.9 & 2 & 6.639576 & 6.94674 & 7.01293 \\
\hline 1 & 2 & 6.638475 & 9.946408 & 7.012778 \\
\hline 0 & 3 & 9.177342 & 9.888691 & 10.05988 \\
\hline 0.1 & 3 & 9.177189 & 9.888635 & 10.05986 \\
\hline 0.3 & 3 & 9.175962 & 9.888215 & 10.05966 \\
\hline 0.5 & 3 & 9.173494 & 9.887381 & 10.05925 \\
\hline 0.7 & 3 & 9.16983 & 9.886127 & 10.05866 \\
\hline 0.9 & 3 & 9.16495 & 9.8834452 & 10.05853 \\
\hline 1 & 3 & 9.162052 & 9.883461 & 10.05806 \\
\hline
\end{tabular}




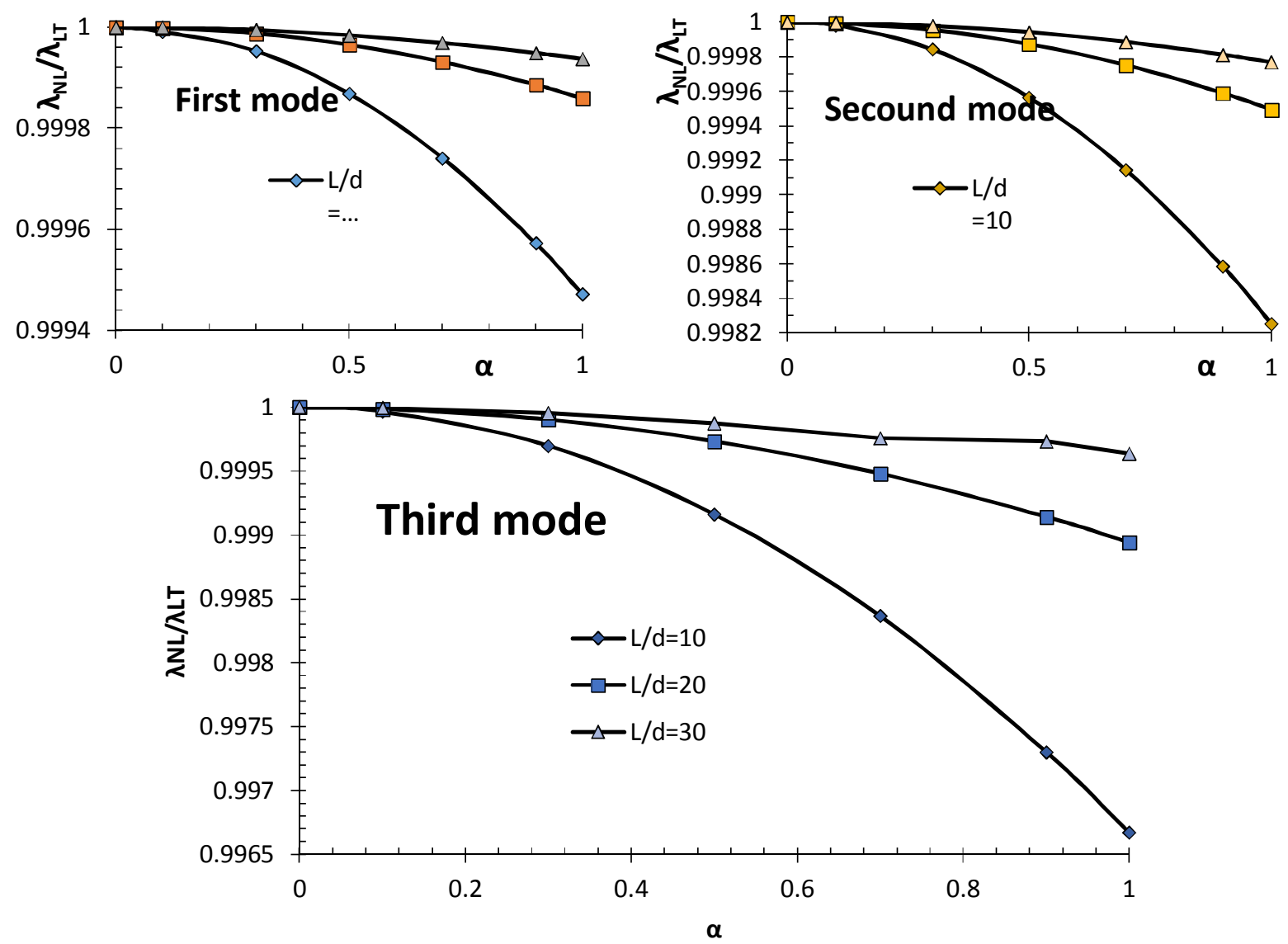

Fig. 6. Small scale effect on first three frequency ratios with different values of $\mathrm{L} / \mathrm{d}$, for Clamped-

Simply supported beam

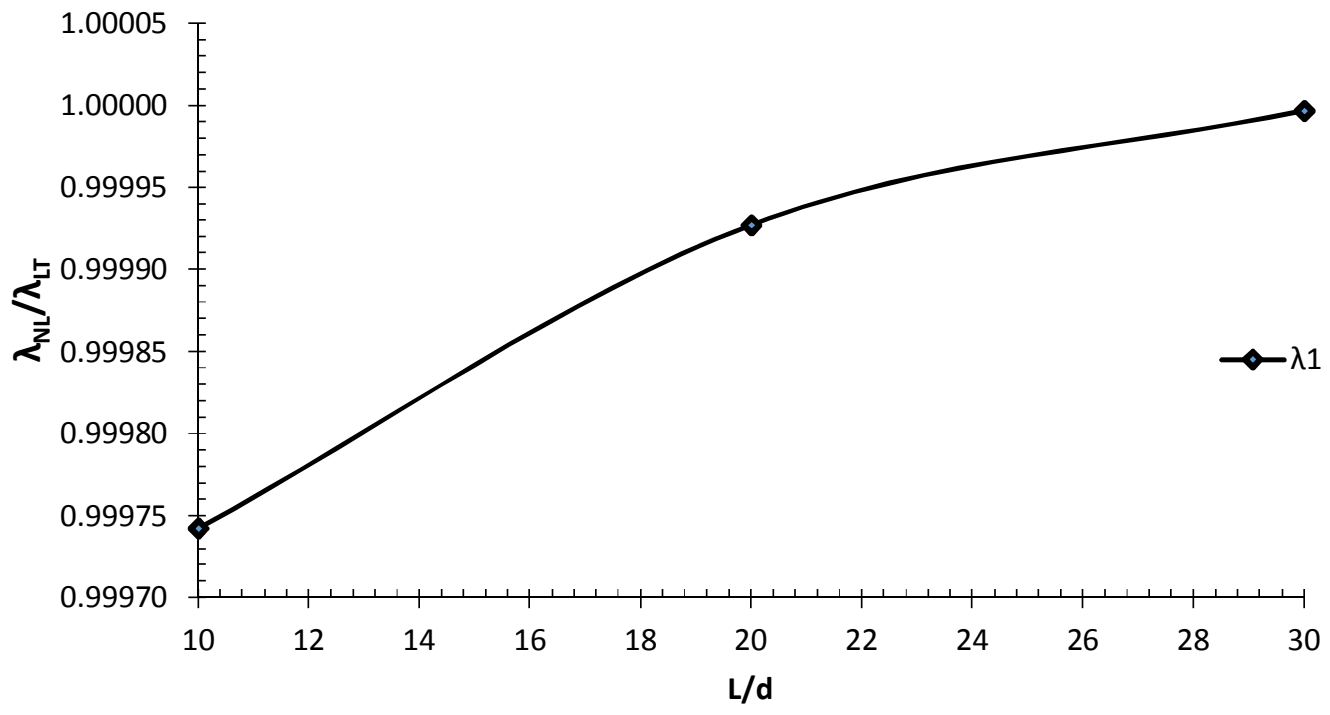

Fig. 7. Effect of slenderness on nonlocal beam frequency, $\alpha=0.7$, both ends clamped 


\section{Conclusions}

A semi-analytical method called differential transformation method is generalized to analyze the vibration characteristics of a SWCNT. The formulation is based on the assumptions of Timoshenko beam theory and the nonlocal differential constitutive relations of Eringen. Unlike the Euler beam model, the Timoshenko beam model allows for the effect of transverse shear deformation and rotary inertia that become significant at short beams and higher frequencies. Also the effect of small scale coefficient and slenderness and various boundary conditions in various frequency ratios are investigated. It is demonstrated that the DTM has high precision and computational efficiency in the vibration analysis of CNTs and nanobeams.

\section{References}

Abdel-Halim Hassan, I. H. (2002). On solving some eigenvalue problems by using a differential transformation. Applied Mathematics and Computation, 127(1), 1-22.

Aifantis, E. C. (1984). On the microstructural origin of certain inelastic models. Journal of Engineering Materials and technology, 106(4), 326-330.

Amara, K., Tounsi, A., Mechab, I., \& Adda-Bedia, E. A. (2010). Nonlocal elasticity effect on column buckling of multiwalled carbon nanotubes under temperature field. Applied Mathematical Modelling, 34(12), 3933-3942.

Ansari, R., \& Sahmani, S. (2012). Small scale effect on vibrational response of single-walled carbon nanotubes with different boundary conditions based on nonlocal beam models. Communications in Nonlinear Science and Numerical Simulation, 17(4), 1965-1979.

Ansari, R., \& Ramezannezhad, H. (2011). Nonlocal Timoshenko beam model for the large-amplitude vibrations of embedded multiwalled carbon nanotubes including thermal effects. Physica E: Lowdimensional Systems and Nanostructures, 43(6), 1171-1178.

Ansari, R., Gholami, R., Hosseini, K., \& Sahmani, S. (2011). A sixth-order compact finite difference method for vibrational analysis of nanobeams embedded in an elastic medium based on nonlocal beam theory. Mathematical and Computer Modelling, 54(11), 2577-2586.

Baughman, R. H., Zakhidov, A. A., \& de Heer, W. A. (2002). Carbon nanotubes--the route toward applications. Science, 297(5582), 787-792.

Chen, C. O. K., \& Ju, S. P. (2004). Application of differential transformation to transient advectivedispersive transport equation. Applied Mathematics and Computation, 155(1), 25-38.

Chow, T. L. (2013). Classical mechanics. CRC Press, Boca Raton, Florida, USA.

Eringen, A. C. (1972a). Linear theory of nonlocal elasticity and dispersion of plane waves. International Journal of Engineering Science, 10(5), 425-435.

Eringen, A. C. (1972b). Nonlocal polar elastic continua. International Journal of Engineering Science, 10(1), 1-16.

Eringen, A. C. (1983). On differential equations of nonlocal elasticity and solutions of screw dislocation and surface waves. Journal of Applied Physics, 54(9), 4703-4710.

Ghorbanpourarani, A., Mohammadimehr, M., Arefmanesh, A., \& Ghasemi, A. (2010). Transverse vibration of short carbon nanotubes using cylindrical shell and beam models. Proceedings of the Institution of Mechanical Engineers, Part C: Journal of Mechanical Engineering Science, 224(3), 745-756.

Iijima, S. (1991). Helical microtubules of graphitic carbon. Nature, 354(6348), 56-58.

Kiani, K., \& Mehri, B. (2010). Assessment of nanotube structures under a moving nanoparticle using nonlocal beam theories. Journal of Sound and Vibration, 329(11), 2241-2264.

Kiani, K. (2010). A meshless approach for free transverse vibration of embedded single-walled nanotubes with arbitrary boundary conditions accounting for nonlocal effect. International Journal of Mechanical Sciences, 52(10), 1343-1356. 
Kiani, K. (2013). Vibration analysis of elastically restrained double-walled carbon nanotubes on elastic foundation subjected to axial load using nonlocal shear deformable beam theories. International Journal of Mechanical Sciences, 68, 16-34.

Liew, K. M., Hu, Y., \& He, X. Q. (2008). Flexural wave propagation in single-walled carbon nanotubes. Journal of Computational and Theoretical Nanoscience, 5(4), 581-586.

Lu, P., Lee, H. P., Lu, C., \& Zhang, P. Q. (2006). Dynamic properties of flexural beams using a nonlocal elasticity model. Journal of Applied Physics, 99(7), 073510.

Maranganti, R., \& Sharma, P. (2007). Length scales at which classical elasticity breaks down for various materials. Physical review letters, 98(19), 195504.

Mindlin, R. D. (1964). Micro-structure in linear elasticity. Archive for Rational Mechanics and Analysis, 16(1), 51-78.

Peddieson, J., Buchanan, G. R., \& McNitt, R. P. (2003). Application of nonlocal continuum models to nanotechnology. International Journal of Engineering Science, 41(3), 305-312.

Samaei, A. T., \& Mirsayar, M. M. (2011). Buckling Analysis of Multi-Walled Carbon Nanotubes with Consideration of Small Scale Effects. Journal of Computational and Theoretical Nanoscience, 8(11), 2214-2219.

Torabi, K., \& Nafar Dastgerdi, J. (2012). An analytical method for free vibration analysis of Timoshenko beam theory applied to cracked nanobeams using a nonlocal elasticity model. Thin Solid Films, 520(21), 6595-6602.

Wang, Z. G. (2013). Axial Vibration Analysis of Stepped Bar by Differential Transformation Method. Applied Mechanics and Materials, 419, 273-279.

Wang, X., \& Cai, H. (2006). Effects of initial stress on non-coaxial resonance of multi-wall carbon nanotubes. Acta materialia, 54(8), 2067-2074.

Wang, C. M., Zhang, Y. Y., \& He, X. Q. (2007). Vibration of nonlocal Timoshenko beams. Nanotechnology, 18(10), 105401.

Wang, Q. (2005). Wave propagation in carbon nanotubes via nonlocal continuum mechanics. Journal of Applied Physics, 98(12), 124301.

Wang, Q., \& Varadan, V. K. (2006). Vibration of carbon nanotubes studied using nonlocal continuum mechanics. Smart Materials and Structures, 15(2), 659.

Wang, C. M., Reddy, J. N., \& Lee, K. H. (Eds.). (2000). Shear deformable beams and plates: Relationships with classical solutions. Elsevier.

$\mathrm{Xu}, \mathrm{M}$. (2006). Free transverse vibrations of nano-to-micron scale beams. Proceedings of the Royal Society A: Mathematical, Physical and Engineering Science, 462(2074), 2977-2995.

Zhang, Y. Q., Liu, G. R., \& Wang, J. S. (2004). Small-scale effects on buckling of multiwalled carbon nanotubes under axial compression. Physical review B, 70(20), 205430.

Zhang, Y. Q., Liu, G. R., \& Xie, X. Y. (2005). Free transverse vibrations of double-walled carbon nanotubes using a theory of nonlocal elasticity. Physical Review B, 71(19), 195404.

Zhu, H., Wang, J., \& Karihaloo, B. (2009). Effects of surface and initial stresses on the bending stiffness of trilayer plates and nanofilms. Journal of Mechanics of Materials and Structures, 4(3), 589-604.

Zhou, J. K. (1986). Differential transformation and its applications for electrical circuits. Huazhong University Press, Wuhan, China. 